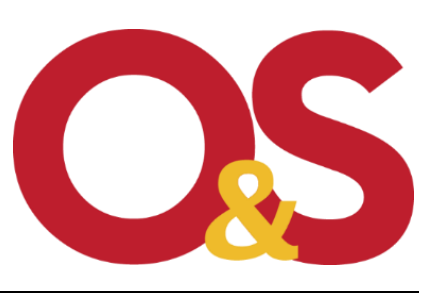

Revista Organizações \& Sociedade 2021, 28(99), 708-720

(C) Autores 2021

Editorial

DOI 10.1590/1984-92302021v28n9900PT

\title{
Impacto Socioambiental da Pesquisa
}

\author{
Andréa Cardoso Ventura ${ }^{a}$ \\ Eduardo Paes Barreto Davel ${ }^{a}$ \\ a Universidade Federal da Bahia, Salvador, Brasil
}

\section{Resumo}

A reflexividade voltada para o futuro do planeta e dos seres que nele habitam é uma questão vital para assegurarmos um amanhã possível. Então, a consideração do impacto socioambiental em toda e qualquer pesquisa deveria se tornar uma preocupação onipresente e incontornável dos(as) pesquisadores(as), das instituições educacionais e das agências reguladoras de pesquisa científica. Isto é, o impacto socioambiental, em suas mais diversas facetas, deve ser incorporado em todo e qualquer processo de geração de conhecimento. Nesse sentido, algumas indagações fomentam essa incorporação na condução da investigação acadêmica: Como a pesquisa pode garantir impactos socioambientais positivos e efetivos à sociedade? Qual é a melhor forma de conduzir a pesquisa para que, desde sua geração, ela produza impactos socioambientais positivos? Como viabilizar a realização de pesquisas totalmente dedicadas à geração de impactos socioambientais positivos, desde a sua realização até a geração de seus resultados? Concluímos que o grau de importância dos impactos socioambientais deve equivaler ou ultrapassar o dos tipos de impactos mais tradicionais, como os socioeconômicos e tecnológicos.

Palavras-chave: impacto; pesquisa; questões socioambientais.

\section{Introdução}

Não conseguimos pensar na vida humana no planeta sem considerar a necessidade de recursos ambientais para sua sobrevivência, de modo que o ser humano impacta a existência do meio ambiente por usá-lo como forma de vida. Historicamente, a cisão entre a natureza e o 
desenvolvimento humano tem origem na Revolução Industrial. Todavia, especialmente nas últimas cinco décadas, já diante da constatação da finitude dos recursos naturais e da incompatibilidade entre o modelo de desenvolvimento existente e a preservação da vida no planeta, a degradação do meio ambiente, as emissões atmosféricas e a poluição da água aumentaram de forma expressiva. Os padrões de consumo e de produção se ampliaram, juntamente com o aumento populacional, agravando a pressão sobre os recursos não renováveis (Soares \& Cassiolato, 2015).

Devido ao acúmulo sistemático de conhecimentos científicos, aos debates internacionais desses conhecimentos e às evidências cada vez mais fartas sobre suas repercussões na vida do planeta, as questões socioambientais se tornaram incontornáveis para a reflexão sobre o futuro dos seres humanos e não humanos. Não é possível pensar em um futuro com prosperidade socioeconômica e tecnológica concomitante às mudanças climáticas globais e catastróficas. Consequentemente, os impactos da pesquisa científica não devem se limitar ou focar nas questões mais tradicionais, como os impactos socioeconômicos e tecnológicos. Ao contrário, os impactos socioambientais devem ser plenamente incorporados em toda e qualquer pesquisa, demonstrando a preocupação de todos(as) os(as) pesquisadores(as) com o futuro do planeta e de sua espécie.

Para melhor entender os impactos socioambientais da pesquisa, abordamos o adjetivo composto pelos termos "social" e "ambiental". Trata-se de um adjetivo amplamente utilizado não apenas no âmbito acadêmico, mas também no organizacional, especialmente quanto a práticas empresariais (Bizerril, Rosa, Carvalho, \& Pedroza, 2015; Cassiolato, Podcameni, \& Soares, 2015; Pessoa, 2021; Teixeira, 2021). Busca-se adotar uma expressão que englobe ações ligadas ao aspecto social que contenham, ao mesmo tempo, traços de preocupação ambiental, assim como ações ligadas à gestão ambiental (Silva Filho, 2007). Áreas do conhecimento distintas, como a sociologia e a ecologia, reconhecem a intrínseca ligação entre questões sociais e ambientais e a denominam de socioambientalismo, ecologismo social, ecologismo popular, ecossocialismo etc. (Martínez Alier, 2007; Viola \& Leis, 2001). No entanto, ainda é necessário muita atenção para garantir a coerência do uso do adjetivo socioambiental, com efetivo equilíbrio entre as suas múltiplas dimensões, especialmente quando se trata de gestão e de responsabilidades (Silva Filho). Não basta que a palavra composta seja utilizada, sem que de fato se observe todas as possíveis interfaces entre as questões sociais e ambientais de cada situação analisada.

Impactos socioambientais podem ser considerados como as alterações sofridas pelo meio ambiente que tenham sido provocadas por determinadas ações ou atividades humanas e que afetam negativamente a qualidade de vida, a saúde, a economia, entre outros aspectos (Diniz, Rodrigues, Sousa, \& Lima, 2020; Hu, 2011; Sumargo, Kasuma, \& Tsang, 2019). Esses impactos podem ser locais, regionais ou globais; podem decorrer de ações ligadas a organizações de diferentes portes e setores produtivos, mas também de indivíduos e comunidades. Apesar de todas as atividades humanas acarretarem efeitos no ambiente, isso ocorre em graus diferenciados, a depender do modo de vida de cada pessoa ou comunidade. Por exemplo, pode-se afirmar que o modo de viver de um indígena causa muito menos impactos aos recursos naturais do planeta do que o de um habitante médio de um país desenvolvido. Essas consequências tendem a ser exponencialmente ampliadas quando o meio ambiente é utilizado para a produção de bens e serviços segundo a lógica predatória do modelo capitalista.

Em âmbito global, as pesquisas sobre os impactos do uso inadequado ou exacerbado dos recursos naturais começam a se destacar a partir da década de 1970 . O conhecimento dos impactos 
que eram (e seguem sendo) produzidos pelas atividades humanas na natureza demandaram uma reflexividade aguçada, uma consciência de futuro e a institucionalização da necessidade de uma gestão ambiental nas esferas global, nacional e local. Apesar disso, a problemática socioambiental contemporânea ainda é tratada de forma secundária, de modo que é concebida em muitas pesquisas como um modismo e permanece "marginalizada do ponto de vista da ação efetiva da sociedade, da política e da gestão" (Pessoa, 2021, p. 6).

As pesquisas que analisam a postura do ser humano frente ao meio ambiente começam a ganhar relevância a partir da primeira grande reunião da Organização das Nações Unidas (ONU) sobre a temática, intitulada de Conferência das Nações Unidas sobre o Meio Ambiente Humano (CNUMAH), em 1972. Considera-se esse momento como a institucionalização da relevância do tema para a sociedade (Pessoa, 2021; Silva Filho, 2007). A Conferência teve como repercussões a criação de diversas outras reuniões globais, tratados internacionais bilaterais ou multipartes, inúmeras regulamentações nacionais e seus consequentes desdobramentos nas normas e regulamentações empresariais. Entretanto, passados quase cinquenta anos dessa institucionalização, a grande maioria dos problemas socioambientais daquele momento (como poluição de solo, água e ar, desmatamentos e extinção de espécies) não só permanecem como se exacerbam (United Nations Framework Convention on Climate Change [UNFCCC], 2020).

O grande debate da atualidade consiste na mudança do clima, que tem causado não apenas o aquecimento global, mas também a intensificação de eventos climáticos extremos, o surgimento de novas doenças, alterações no regime das chuvas, dificuldades de produção de alimentos, migrações em massa, entre outros (ONU, 2015; Ventura, Fernández García, \& Andrade, 2019). Muitos consideram que a crise causada pela pandemia de Covid-19 é apenas um teste para o que ainda será enfrentado pela humanidade com a questão climática, motivo pelo qual deve ser utilizada para aprendizados relativos à cooperação entre atores de diversas esferas, para a coordenação de ações globais-locais etc. (Silva, Soares, Machado, \& Arbilla, 2020).

Por um lado, verifica-se que as questões socioambientais atingem todas as escalas geográficas, global, nacional e local. Por outro lado, seus impactos são marcadamente mais intensos sobre as populações pobres e discriminadas, a exemplo das populações indígenas e negras, dentre outros grupos sociais marginalizados (Acserald, 2010; Martínez Alier, 2007), o que requer mudanças efetivas na relação entre sociedade e ambiente. Os problemas socioambientais tendem a reforçar a desigualdade social, visto que afetam primeiramente os países e segmentos da população mundial mais pobres, cujas múltiplas vulnerabilidades fazem com que sofram mais fortemente as repercussões adversas de catástrofes naturais. Consequentemente, o risco de crescentes tensões e conflitos ligados à problemática socioambiental é cada vez mais intenso em todo o mundo (Soares \& Cassiolato, 2015).

A partir de pesquisas realizadas na década de 1970, Herman Daly, economista estadunidense, contribui para a construção de um novo paradigma científico: a economia ecológica. Esse paradigma compreende a definição de limites biofísicos para o uso econômico dos recursos naturais, a fim de evitar a exploração desenfreada e prejudicial ao meio ambiente. Posteriormente, o pesquisador torna-se economista-chefe do Banco Mundial, função que o tornou um dos responsáveis pela elaboração dos princípios políticos básicos que viriam a conformar o chamado desenvolvimento sustentável (Daly, 1999). É justamente a evolução dessas discussões que culmina na publicação, em 1987, do relatório Nosso Futuro Comum (United Nations, 1987), considerado o 
grande marco para a consolidação da noção de desenvolvimento sustentável. Essa publicação integra aspectos que iriam além do desenvolvimento econômico, valorizando, assim, a proteção ao meio ambiente e a busca por equidade social. Observa-se que a noção da "nova" proposta de modelo de desenvolvimento já considera a indissociabilidade entre as questões sociais e ambientais. Entretanto, "o discurso de desenvolvimento sustentável não foi capaz de produzir políticas abrangentes nem mudanças radicais no comportamento individual e coletivo que são imprescindíveis em escala global" (Soares \& Cassiolato, 2015, p. 178).

As alternativas de enfrentamento das questões socioambientais são produzidas pela ciência (Pessoa, 2021; Soares \& Cassiolato, 2015) e exigem a atuação urgente de variados setores da sociedade, como Estado, empresas e sociedade civil (Teixeira, 2021). Assim, a pesquisa científica assume papel de total relevância a fim de contribuir não apenas com a criação de capacidades tecnológicas relacionadas à atual dependência de combustíveis fósseis e outros recursos não renováveis. Ela deverá também contribuir com a geração de um novo modo de conhecimento que permita que preocupações socioambientais assumam posição de efetivo protagonismo frente a toda e qualquer decisão a ser tomada pelo futuro da humanidade.

É necessário que todos os campos do conhecimento busquem produzir soluções para as complexas questões que se nos apresentam. É preciso mais do que soluções tecnológicas que viabilizem o desenvolvimento de produtos ou a oferta de serviços menos dependentes de recursos naturais, seja na área pública, privada ou do terceiro setor. Ao mesmo tempo, é premente que essas resoluções cheguem a resultados positivos que contribuam com a minimização das graves desigualdades sociais que afligem nossa sociedade, com menor impacto possível ao meio ambiente. É necessário que a pesquisa considere as necessidades das populações mais vulneráveis, analisando todos os aspectos a elas relacionados.

\section{Geração de impactos socioambientais positivos: uma preocupação da pesquisa científica}

Há urgência de que a agenda socioambiental seja incorporada nas discussões dos mais diversos atores sociais de diferentes países. A velocidade dessa incorporação está diretamente ligada à ênfase dada a essas questões pela comunidade científica (Silva Filho, 2007). Assim como ocorreu na década de 1970, quando as pesquisas desencadearam toda uma nova agenda de preocupações para empresas, governos e outros setores da sociedade, em prol de novas atitudes frente a questões socioambientais, acreditamos que, na atualidade, a situação precisa se repetir com maior ênfase. Tanto a natureza quanto a sociedade demonstram que a forma como o modelo de desenvolvimento está sendo empregado não resulta em efeitos socioambientais positivos, como confirmam as mudanças climáticas e até mesmo a pandemia de Covid-19. Alguns países, inúmeras empresas e múltiplos organismos da sociedade civil buscam realizar transformações em sua forma de agir. Todavia, a ciência, por meio da pesquisa, pode acelerar fortemente esse processo, que ainda é tímido e insuficiente frente às necessidades concretas.

Há um reconhecimento crescente de que essa problemática somente será resolvida por meio do uso de conhecimentos interdisciplinares que também incorporem diálogos de saberes entre a academia e povos tradicionais, por exemplo. Como relembra Pessoa (2021), essa proposta tem sido advogada por grandes cientistas sociais contemporâneos, como Edgar Morin, Enrique Leff 
e Boaventura de Souza Santos. Urge a construção de novas formas de racionalidade na relação entre sociedade e natureza. Até o momento atual, a grande maioria das constatações científicas publicadas demonstram o efeito potencial de a humanidade modificar e explorar a natureza sem medir as consequências dos impactos ambientais (Andrade \& Oliveira, 2021).

Nesse contexto, destaca-se o papel das instituições de ensino superior e de pesquisa, organizações detentoras de importante papel na sociedade enquanto formadoras de competências e análise crítica. É inegável a capacidade instalada nessas instituições para a criação de um ambiente capaz de fomentar a formação de lideranças e propor discussões, soluções e tecnologias que contribuem para que a própria sociedade possa lidar com os seus desafios (Bizerril et al., 2015; Bolan \& Motta, 2007; Lacerda, Silva, Souza, \& Lira, 2014). Dentre os papéis da universidade, destaca-se a promoção de pesquisa interdisciplinar e de conhecimento científico, voltada à resolução de problemas da sociedade (Venzke \& Nascimento, 2013).

A discussão sobre o papel dos sistemas educacionais na geração de conhecimento em busca da solução de problemas socioambientais é tema de debates internacionais promovidos pela ONU. A década de 2005 a 2014 foi instituída como a Década da Educação para o Desenvolvimento Sustentável (DEDS). No momento, a temática consta entre os Objetivos do Desenvolvimento Sustentável (ODS), ligados à Agenda 2030 para o desenvolvimento (ONU, 2015). Em síntese, os ODS incluem um complexo conjunto de desafios em diversas áreas (econômicas, sociais e ambientais), que requerem transformações na estrutura e no funcionamento das sociedades. A educação é considerada ferramenta essencial no alcance deste desafio, pois é impossível negar o papel estrutural da universidade como um dos principais atores diante das mudanças e objetivos pretendidos (Serafini, Moura, \& Rezende, 2020; Teferra, 2020). Se o papel da universidade é a produção indissociável de ensino, pesquisa e extensão, a pesquisa terá função inequívoca nesse sentido.

Entretanto, para que essa atribuição seja bem cumprida, é necessário que as pesquisas se afastem da abordagem marcadamente antropocênica. As questões socioambientais devem ser promovidas a partir de uma visão integral, e não de maneira marginal (Barbieri \& Silva, 2011; Jacobi, Raufflet, \& Arruda, 2011; Venzke \& Nascimento, 2013). Nesse sentido, Miller, Muñoz-Erickson e Redman (2011) defendem a adoção de pesquisas que garantam o pluralismo epistemológico e reflexividade, como elementos necessários para a produção de elementos-chave na transição para a sustentabilidade. O pluralismo epistemológico, já mencionado anteriormente na necessidade de interdisciplinaridade e diálogo de saberes, baseia-se no reconhecimento e na combinação de múltiplas formas de conhecimento. É necessário que as pesquisas lancem mão de todos os conhecimentos relevantes, assim como perspectivas e pontos de vista diferenciados, a fim de garantir uma compreensão mais diversa, ampla e fina da questão eminente que enfrentamos e que clama por soluções efetivas.

\section{Impactos socioambientais na pesquisa em administração}

Nesse contexto, o papel das universidades que trabalham com a formação em administração torna-se mais relevante e estratégico. Conforme indicado nas próprias Diretrizes Curriculares Nacionais do Curso de Graduação em Administração (Brasil, 2005), essa formação deve estar comprometida com a melhoria das condições humanas. Portanto, as pesquisas desenvolvidas no 
campo da gestão devem desviar-se totalmente das características apresentadas na análise de Horkheimer (1990), que as considerava fortemente ligadas ao pragmatismo e ao sucesso de mercado. Aparentemente, essas pesquisas ainda enfatizam excessivamente o papel dos gestores para favorecer o mercado (Demajorovic \& Silva, 2012; Jacobi et al., 2011; Venzke \& Nascimento, 2013). É necessária uma virada de mentalidade para que se busque fazer frente aos desafios que a humanidade já enfrenta e ainda enfrentará. Por outro lado, as escolas de administração que mais se destacam internacionalmente, posicionadas como relevantes e inovadoras, são aquelas que se orientam para considerar plenamente as questões socioambientais como competências da prática de gestão que prima pelo futuro sustentável do planeta e das pessoas (Muff et al., 2013).

Mesmo que a gestão sustentável de recursos naturais e do futuro seja um campo interdisciplinar do conhecimento (Chiras \& Reganold, 2009; Robertson, 2021), a preocupação com as questões socioambientais e sua gestão torna-se um subcampo de pesquisas na área de administração, ao mesmo tempo em que se incorpora a várias outras temáticas. Por exemplo, há pesquisas que, a partir da sustentabilidade, abordam o comportamento (Lane \& Maznevski, 2019; Pinheiro, Machado, Nascimento, Peñaloza, \& Pinheiro, 2020), a gestão de projetos (Silvius, Schipper, Planko, \& Van Den Brink, 2012) e a liderança (Hull, Robertson, \& Mortimer, 2020; Mackey, Mclntosh, \& Phipps, 2020; Ritz \& Rimanoczy, 2021).

No entanto, para além da incorporação das questões socioambientais como subcampo de pesquisas em administração, a gestão socioambiental também tem ganhado força como um campo de pesquisa em si mesmo. Ela tem sido abordada, por exemplo, com enfoque em questões de planejamento (Lima, Lima Júnior, Assis, \& Gurgel, 2018) e de tomada de decisão (Silva \& Razzolini Filho, 2021). Na área da gestão privada, é crescente o número de investigações que abordam questões relativas a mudanças organizacionais frente à temática (Coelho, Fragoso, Santiago, \& Pinheiro, 2020; Pinsky \& Kruglianskas, 2017) e seu impacto na avaliação de riscos e investimentos (Torinelli \& Silva Júnior, 2021). Quanto à administração pública, os trabalhos enfocam cada vez mais a eficiência da gestão sustentável nesse setor (Soares \& Gomes, 2017), de modo que a adoção da Agenda Ambiental da Administração Pública (A3P) (Lanzarin, Camargo, Mazzioni, \& Zanin, 2018) figura como uma de suas estratégias. Também se destaca o papel da temática no desenvolvimento de cidades (Faria, Russi, Marcato, \& Paschoalin Filho, 2017; Leão, Andrade, \& Nascimento, 2021) e até mesmo no desenvolvimento de políticas públicas (Góes, Andrade, Jabbour, \& Silva, 2021), especialmente frente às mudanças climáticas. Esse breve apanhado de exemplificações sobre as pesquisas que têm sido realizadas na interface entre as questões socioambientais e a administração não passam de um extrato da enorme e variada gama de conhecimentos necessários e relevantes.

\section{Como incluir os impactos socioambientais na prática da pesquisa?}

Incluir os impactos socioambientais na pesquisa acadêmica pressupõe reconhecer que tudo o quanto se desenvolve para indivíduos e organizações terá impactos, em maior ou menor grau, sobre aspectos ligados à natureza e à vida humana. Se toda e qualquer atividade humana é circunscrita aos limites físicos impostos pela finitude dos recursos naturais, e se o modo de vida de cada pessoa ou comunidade impacta esses recursos de maneira diferenciada, é imprescindível que isso seja considerado ao longo de todo o processo de pesquisa, desde sua concepção, execução e composição. 
A problemática socioambiental exige uma mudança de paradigma (Fernandes \& Sampaio, 2008) e de mentalidade que se sustentaria em uma racionalidade alternativa aos grandes conflitos da sociedade moderna. São conflitos que podem ser resumidos na difícil relação entre desenvolvimento econômico e preservação ambiental. Há evidências crescentes do esgotamento do modelo produtivo baseado na exploração intensiva de recursos naturais, especialmente os não renováveis (Cassiolato, et al., 2015). Por essa razão, além de o novo modelo produtivo ter que ser centrado numa economia de baixo carbono e em recursos energéticos renováveis, há premência da construção de um novo arcabouço de políticas de inovação para o desenvolvimento social e ambientalmente sustentável. Assim, essa mudança passa pela inserção da sustentabilidade socioambiental, em todas as suas dimensões, como critério de desenvolvimento. Para que isso ocorra, é necessário o apoio da ciência, por meio de pesquisas que rompam com o determinismo epistemológico, que incorporem a atuação interdisciplinar e que permitam o diálogo de saberes.

Não há como atingir uma nova situação socioambiental sem ciência, tecnologia e inovação. Esta deve ser vista como um fator facilitador que possibilite a abertura de um novo conjunto de soluções para o enfrentamento das necessidades de sociedades (Chesnais, 2015). Porém, a adoção de novos sistemas inovativos não depende apenas do desempenho das empresas e de organizações de ensino e pesquisa (Cassiolato et al., 2015). É necessário observar como esses elementos do sistema interagem entre si e com vários outros atores sociais e instituições, inclusive as políticas. Assim, fica evidenciado que, para que haja impacto socioambiental positivo, não bastam pesquisas ligadas ao desenvolvimento de produtos e serviços menos agressivos ao meio ambiente. Diversas pesquisas nas áreas de ciências humanas e sociais aplicadas, por exemplo, são necessárias para auxiliar na compreensão tanto dos fatores dificultadores da inovação voltada à solução das necessidades humanas quanto das possibilidades existentes.

As possibilidades a serem analisadas devem ultrapassar as chamadas inovações verdes, consideradas como aquelas que permitem ganhos de eficiência com cuidados ao meio ambiente. Isso porque a maioria delas não leva em consideração as contradições inerentes à dinâmica capitalista, baseada no crescimento desigual e ilimitado. Os ganhos de eficiência contidos na lógica do "crescimento verde", por si só, não são suficientes para resolver a escassez de recursos naturais, superar os limites ecológicos e reduzir as grandes problemáticas que entrelaçam questões ambientais e sociais (Hoffmann, 2011). A escala requerida para reduzir as mudanças climáticas, principal e mais complexa problemática enfrentada pela humanidade atualmente, é tão grande que inovações tradicionais não serão suficientes para dar respostas eficazes e capazes de garantir, ao mesmo tempo, a preservação da atmosfera e o desenvolvimento de países emergentes e populações marginalizadas (Hoffmann).

Provavelmente, a ótica da inovação social é o caminho para que as soluções que venham a ser desenvolvidas com base na preocupação com impactos socioambientais sejam de fato capazes de provocar rupturas que gerem mudanças sociais profundamente transformadoras. Pode-se definir inovação social como atividades e serviços inovadores que são motivados pelo objetivo de satisfazer uma necessidade social, de modo que são predominantemente difundidos por meio de organizações com propósito social (Mulgan, 2006). Ou seja, trata-se de inovações cujos esforços são canalizados, especialmente, para a solução das questões que afligem a humanidade (Murray, Caulier-Grice, \& Mulgan, 2010). As discussões sobre esse tipo específico de inovação receberam 
destaque especial na última década, sendo consideradas como uma alternativa às crescentes pressões de questões socioambientais.

São muitas as tentativas de Estados para a incorporação da consideração dos impactos socioambientais em iniciativas voltadas ao desenvolvimento: (a) propostas europeias do chamado "Green New Deal", (b) estratégias de resiliência propostas por cidades integrantes da rede 100RC, que integra 100 cidades que buscam seu fortalecimento e preparação frente aos desafios sociais e climáticos existentes, etc. Essas soluções têm sido estimuladas pelo mercado, por meio de novos investimentos verdes, abarcados na sigla ESG (Environmental and Social Governance), o que demonstra que os investidores estão focando seus recursos em organizações que não só garantam o cumprimento de requisitos básicos ligados às áreas de meio ambiente e de fatores sociais, como também que sejam pautadas por uma governança transparente e ética.

Certamente, essas novas demandas de atores sociais como governos, empresas e o próprio mercado financeiro trazem consigo a premência da construção de novos conhecimentos. Para conseguirmos assegurar o futuro do planeta e dos seres que nele habitam, continua sendo cada vez mais imperioso o desenvolvimento de pesquisas que viabilizem mudanças de visões, de comportamentos, de políticas públicas e de práticas gerenciais. Há décadas já se fala sobre a necessidade de uma mudança comportamental, assim como de medidas de enfrentamento, que advirão de diversos atores sociais, entre eles os cientistas, para reorientar os modelos de desenvolvimento baseado exclusivamente na mercantilização da natureza. O momento urge por soluções efetivas, que façam das questões socioambientais um critério de impacto da pesquisa científica de forma generalizada e ampla, transformando essa preocupação em uma causa de todos em prol do futuro destas e das próximas gerações.

\section{Referências}

Acserald, H. (2010). Ambientalização das lutas sociais: o caso do movimento por justiça ambiental. Estudos Avançados, 24(68), 103-119. doi:10.1590/S0103-40142010000100010

Andrade, K., \& Oliveira, J. G. B. (2021). Educação ambiental: estratégias de conscientização ou sensibilização sobre as ações antrópicas no Brasil? In R. L. P. Teixeira (Org.), Problemas socioambientais emergentes: Contribuições teóricas e práticas (pp. 13-21). Bauru, SP: Gradus.

Barbieri, J. C., \& Silva, D. (2011). Educação ambiental na formação do administrador. São Paulo, SP: Cengage Learning.

Bizerril, M., Rosa, M. J., Carvalho, T., \& Pedrosa, J. (2015). A sustentabilidade socioambiental no ensino superior: Um tema integrador para os países de língua portuguesa? Revista Forges, 2(1), 99-115.

Bolan, V., \& Motta, M. V. (2007). Responsabilidade social no ensino Superior. Revista de Educação, 10(10), 204-2010. Recuperado de https://bit.ly/3AkeSiB

Cassiolato, J. E., Podcameni, M. G., \& Soares, M. C. (2015). Sustentabilidade socioambiental em um contexto de crise: uma introdução. In J. E. Cassiolato, M. G. Podcameni, \& M. C. Soares (Orgs.), Sustentabilidade socioambiental em um contexto de crise (pp. 21-39). Rio de Janeiro, RJ: Epapers. 
Chesnais, F. (2015). Uma interpretação sobre a situação econômica mundial seguida por considerações sobre a crise ambiental. In J. E. Cassiolato, M. G. Podcameni, \& M. C. Soares (Orgs.), Sustentabilidade socioambiental em um contexto de crise (pp. 39-65). Rio de Janeiro, RJ: Epapers.

Chiras, D., \& Reganold, J. (2009). Natural resource conservation: Management for a sustainable future. Londres: Pearson.

Coelho, M. B., Fragoso, E. J. N., Santiago, A. M. S, \& Pinheiro, F. A. (2020). Gestão de pessoas e sustentabilidade: Aspectos empíricos e práticos. Revista Multidisciplinar de Psicologia, 14(51), 349-365. doi:10.14295/idonline.v14i51.2582

Daly, H. (1999). Ecological economics and the ecology of economics: essays in criticism. Cheltenham: Edward Elgar Press.

Demajorovic, J., \& Silva, H. C. O. (2012). Formação interdisciplinar e sustentabilidade em cursos de administração: Desafios e perspectivas. Revista de Administração Mackenzie, 13(5), 39-64. doi:10.1590/S1678-69712012000500003

Diniz, A. N., Rodrigues, C. F., Sousa, M. A., \& Lima, E. C. (2020). Diagnóstico dos impactos socioambientais da Serra da Meruoca-CE. Homem, Espaço e Tempo, 14(3), 127-141.

Faria, A. C., Russi, R. A., Marcato, A. F. G., \& Paschoalin Filho, J. A. (2017). Sustentabilidade urbana e o desenvolvimento de São Francisco (EUA). Desenvolvimento Regional em Debate, 7(1), 214-235. doi:10.24302/drd.v1i1.1406

Fernandes, V., \& Sampaio, C. A. C. (2008). Problemática ambiental ou problemática socioambiental? A natureza da relação sociedade/meio ambiente. Desenvolvimento e Meio Ambiente, 18, 87-94. doi:10.5380/dma.v18i0.13427

Góes, M. F. B., Andrade, J. C. S., Jabbour, C. J. C., \& Silva, M. S. (2021). Wind power projects in Brazil: challenges and opportunities increasing co-benefits and implications for climate and energy policies. Environment, Development and Sustainability, 23, 1534115367.doi:10.1007/s10668-021-01300-8

Hoffmann, U. (2011). Some reflections on climate change, green growth illusions and development space. Genebra: Unctad.

Horkheimer, M. (1990). Teoria crítica: Uma documentação (H. Cohn, Trad.). São Paulo, SP: Perspectiva.

$\mathrm{Hu}, \mathrm{C.}$ K. (2011). Evaluation of social environment impact of highway construction using gray matter-element information entropy model. Journal of Environmental Science and Engineering, 5(12), 130-136.

Hull, R. B., Robertson, D. P., \& Mortimer, M. (2020). Leadership for sustainability: Strategies for tackling wicked problems. Washington, DC: Island Press.

Jacobi, P. R., Raufflet, E., \& Arruda, M. P. (2011). Educação para a sustentabilidade nos cursos de administração: Reflexão sobre paradigmas e práticas. Revista de Administração Mackenzie, 12(3), 21-50. doi:10.1590/S1678-69712011000300003

Lane, H. W., \& Maznevski, M. L. (2019). International management behavior: Global and 
sustainable leadership. Cambridge: Cambridge University Press.

Lanzarin, J., Camargo, T. F., Mazzioni, S., \& Zanin, A. (2018). Agenda ambiental da administração pública em instituições federais de ensino superior. Brazilian Journal of Development, 4(3), 1020-1044. doi:10.34117/bjdv4n3-166

Lacerda, C. C. O., Silva, N. E. F., Souza, S. M. A., \& Lira, W. S. (2014). Ações de marketing social desenvolvidas pelas instituições de ensino superior privadas na cidade de Campina Grande-PB. Artigo apresentado no XVI Encontro Nacional de Gestão e Meio Ambiente, São Paulo, SP. Recuperado de https://bit.ly/2VO2Q1Z

Leão, E. B. S., Andrade, J. C. S., \& Nascimento, L. F. (2021). Recife: A climate action profile. Cities, 116, 103270. doi:10.1016/j.cities.2021.103270

Lima, L. J., Lima Júnior, J. F., Assis, R. R. Q., \& Gurgel, G. C. D. L. (2018). Planejamento institucional em Saúde: O caso da sustentabilidade ambiental em um hospital. Revista de Pesquisa Interdisciplinar, 3(1), 2-14. doi:10.24219/rpi.v3i1.248

Mackey, J., McIntosh, P., \& Phipps, C. (2020). Conscious Leadership: Elevating humanity through business. Nova lorque: Portfolio.

Martínez Alier, J. (2007). O ecologismo dos pobres: Conflitos ambientais e linguagens de valoração. São Paulo, SP: Contexto.

Miller, T. R., Muñoz-Erickson, T., Redman, C. L. (2011). Transforming knowledge for sustainability: Towards adaptive academic institutions. International Journal of Sustainability in Higher Education, 12(2), 177-192. doi:10.1108/14676371111118228

Muff, K., Dyllick, T., Drewell, M., North, J., Shrivastava, P., \& Haertle, J. (2013). Management education for the world: a vision for business schools serving people and planet. Cheltenham: Edward Elgar.

Mulgan, G. (2006). The process of social innovation. Innovations: Technology, Governance, Globalization, 1(2), 145-162. doi:10.1162/itgg.2006.1.2.145

Murray, R., Caulier-Grice, J., \& Mulgan, G. (2010). The open book of social innovation. Londres: Young Foundation. Recuperado de https://bit.ly/2VHpPf5

Organização das Nações Unidas. (2015). Transformando Nosso Mundo: a Agenda 2030 para o Desenvolvimento Sustentável (Centro de Informação das Nações Unidas para o Brasil, Trad.). New York: UNDP. Recuperado de https://bit.ly/3AK218M

Pessoa, Z. S. (2021). Prefácio. In R. L. P. Teixeira (Org.), Problemas socioambientais emergentes: Contribuições teóricas e práticas (pp. 7-9). Bauru, SP: Gradus.

Pinheiro, L. V. S., Machado, D. Q., Nascimento, J. C. H. B., Peñaloza, V., \& Pinheiro, J. (2020). Comportamento ecológico em tempos de (in)sustentabilidade. Faces Journal, 19(3), 8-25. Recuperado de https://bit.ly/39hXAHg

Pinsky, V., \& Kruglianskas, I. (2017). Inovação tecnológica para a sustentabilidade. Estudos Avançados, 31(90), 107-126. doi:10.1590/s0103-40142017.3190008

Resolução no 4, de 13 de julho de 2005. (2005). Institui as Diretrizes Curriculares Nacionais do 
Curso de Graduação em Administração, bacharelado, e dá outras providências. Diário Oficial da União. Recuperado de https://bit.ly/3hTK5IR

Ritz, A., \& Rimanoczy, I. (Eds.). (2021). Sustainability mindset and transformative leadership: A multidisciplinary perspective. Londres: Palgrave Macmillan.

Robertson, M. (2021). Sustainability principles and practice. Londres: Routledge.

Serafini, P. G., Moura, J. M., \& Rezende, J. F. D. (2020). Observing sustainable development goals in Brazilian federal universities. Artigo apresentado na 8a International Conference on Sustainable Development, Nova lorque.

Silva, C. M., Soares, R., Machado, W., \& Arbilla, G. (2020). A pandemia de COVID-19: Vivendo no antropoceno. Revista Virtual de Química, 12(4), 1001-1016. doi:10.21577/19846835.20200081

Silva Filho, J. C. L. (2007). Socioambiental: O perigo da diluição de dois conceitos. Revista Eletrônica de Gestão Organizacional, 5(2), 198-209.

Silva, R. F., \& Razzolini Filho, E. (2021). O papel da informação sobre sustentabilidade nos processos de tomada de decisão. Revista Metropolitana de Sustentabilidade, 11(1).

Silvius, G., Schipper, R., Planko, J., \& Van Den Brink, J. (2012). Sustainability in project management. Londres: Routledge.

Soares, I. J., \& Gomes, M. F. (2017). O princípio da eficiência sustentável na Administração Pública. Revista de Direito e Sustentabilidade, 3(1), 72-90. doi:10.26668/IndexLawJournals/25259687/2017.v3i1.1992

Soares, M. C., \& Cassiolato, J. E. (2015). Crise, sustentabilidade e mudança tecnológica. In J. E Cassiolato, M. G. Podcameni, \& M. C. Soares, Sustentabilidade socioambiental em um contexto de crise (pp. 173-192). Rio de Janeiro, RJ: Epapers.

Sumargo, B., Kasuma, K. A. P., \& Tsang, Y. F. (2019). Social-environment factor as a weak point of sustainable development in Indonesia. AIP Conference Proceedings, 2019(1). doi:10.1063/1.5061866

Teferra, D. (2020). O ensino superior e os ODS na África: mais do mesmo? International Higher Education, 100, 15-17.

Teixeira, R. L. P. (Org.). (2021). Problemas socioambientais emergentes: Contribuições teóricas e práticas. Bauru, SP: Gradus.

Torinelli, V. H., \& Silva Júnior, A. F. A. (2021). Environmental risk analysis (ERA) in the strategic asset allocation (SAA) of the international reserves (IRs) managed by central banks (CBs). Latin American Journal of Central Banking, 2(1), 1-17. doi:10.1016/j.latcb.2021.100021

United Nations. (1987). Report of the World Commission on Environmental and Development: Our Common Future. Genebra: WCED.

United Nations Framework Convention on Climate Change. (2020). United Nations Climate Change Annual Report 2020. Bonn: UNFCCC. Recuperado de https://bit.ly/3A42HGz

Ventura, A. C., Fernández Garcia, L. F., \& Andrade, J. C. S. (2019). O potencial das tecnologias 
sociais de convivência com o semiárido para a geração de sinergia entre mitigação e adaptação às mudanças climáticas: Um caso ilustrativo. Revista Econômica do Nordeste, 50(1), 65-83. Recuperado de https://bit.ly/39druft

Venzke, C. S., \& Nascimento, L. F. M. (2013). Caminhos e desafios para a inserção da sustentabilidade socioambiental na formação do administrador brasileiro. Revista de Administração da Mackenzie, 14(3), 26-54. doi:10.1590/S1678-69712013000300003

Viola, E. J., \& Leis, H. R. (2001). O ambientalismo multisetorial no Brasil para além da Rio-92: O desafio de uma estratégia globalista viável. In E. J. Viola, H. R. Leis, I. Scherer-Warren, J. S. Guivant, P. F. Vieira, \& P. J. Krischke, Meio ambiente, desenvolvimento e cidadania: Desafios para as ciências sociais (pp. 134-160). São Paulo: Cortez.

\section{Autoria}

\section{Andréa Cardoso Ventura}

Doutora com pós-doutorado em administração pela Escola de Administração da Universidade Federal da Bahia. Professora da Escola de Administração da Universidade Federal da Bahia. Coordenadora do grupo de pesquisa Governança para Sustentabilidade e Gestão de Baixo Carbono. Coordenadora da câmara temática de Resiliência Urbana do Painel Salvador de Mudança do Clima. Editora associada da revista Organizações \& Sociedade para o eixo "Organizações, Gestão Ambiental e Sustentabilidade". Pesquisa e publica sobre mudanças climáticas, tecnologias sociais e resiliência climática e urbana.

E-mail: andreaventurassa@gmail.com

ORCID: https://orcid.org/0000-0002-4371-632X

\section{Eduardo Paes Barreto Davel}

Doutor em administração pela École des Hautes Études Commerciales de Montréal (Canadá), com pós-doutorado em administração pela Nova School of Business and Economics da Universidade Nova de Lisboa (Portugal). Professor na Escola de Administração da Universidade Federal da Bahia. Editor-chefe da revista Organizações \& Sociedade. Pesquisa e publica sobre empreendedorismo cultural, gestão do processo criativo, ensino, metodologia, aprendizagem, cultura e estética nas organizações.

E-mail: davel.eduardo@gmail.com

ORCID: https://orcid.org/0000-0003-0610-6474

\section{Contribuição dos autores}

Primeira autora: concepção (igual), investigação (igual), redação - rascunho original (igual).

Segundo autor: concepção (igual), investigação (igual), redação - rascunho original (igual). 
A O\&S é signatária do DORA (The Declaration on Research Assessment) e do COPE (Committee on Publication Ethics).

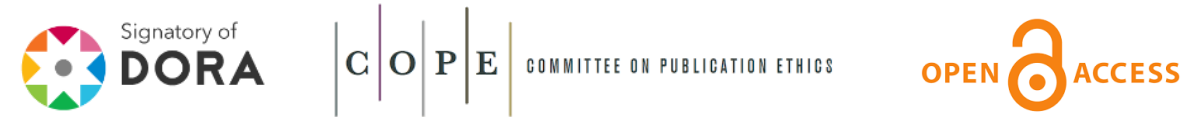

(c) (i) 